\title{
CYP2J2-Derived EETs Attenuated Angiotensin II-Induced Adventitial Remodeling via Reduced Inflammatory Response
}

\author{
Chi Zhou ${ }^{\mathrm{a}}$ Jin Huanga Junxiong Chen ${ }^{\mathrm{a}}$ Jinsheng Laia Fasheng Zhu ${ }^{\mathrm{a}}$ Xizhen $\mathrm{Xu}^{\mathrm{a}}$ \\ Dao Wen Wang ${ }^{a}$
}

a Department of Internal Medicine and Institute of Hypertension, Tongji Hospital, Tongji Medical College of Huazhong University of Science and Technology, Wuhan, China

\section{Key Words}

Cytochrome P450 epoxygenase 2J2 - Epoxyeicosatrienoic acids - Adventitial remodeling • Angiotensin II • PPARY

\begin{abstract}
Background: Cytochrome P450 (CYP) epoxygenases metabolize arachidonic acids (AA) to form epoxyeicosatrienoic acids (EETs), which exert beneficial roles in the treatment of cardiovascular diseases, but little is known about its role on adventitial remodeling. Methods: We used C57BL/6J mice in vivo and primary rat adventitial fibroblasts (AFs) in vitro treated with Angiotensin II to investigate the effects of CYP2J2 gene delivery and exogenous EETs administration on adventitial remodeling. Results: CYP/sEH system was found to exist in human adventitia, and involved in adventitial remodeling process. Exogenous EETs administration significantly inhibited Ang II-induced AFs activation, characterized by differentiation, proliferation, migration, and collagen synthesis. These protective effects were partially reversed by PPAR $\gamma$ antagonist GW9662 pretreatment or SOCS3 siRNA transfection. EETs suppressed Ang II-induced IKB $\alpha$ phosphorylation, subsequent NF-KB nuclear translocation via PPARY dependent signaling pathway in AFs. Additionally, EETs reduced Ang II-induced JAK2, STAT3 phosphorylation and subsequent phosphor-STAT3 nuclear translocation, which were mediated by SOCS3 induction but independent of PPARY activation. Furthermore, rAAV-CYP2J2 gene delivery reduced vessel wall thickening, AFs differentiation, proliferation and collagen deposition in aortic adventitia induced by Ang II infusion, which were mediated by NF-KB and SOCS3/JAK/STAT signaling pathways in blood pressure dependent and independent manner, respectively. Conclusion: We concluded that CYP2J2 overexpression attenuated Ang II-induced adventitial remodeling via PPAR $\gamma$-dependent NF-KB and PPAR $\gamma$-independent SOCS3/JAK/STAT inflammatory signaling pathways.
\end{abstract}

Dao Wen Wang, M.D., Ph.D. and Xizhen Xu, M.D., Ph.D.

\section{KARGER}

Department of Internal Medicine and Institute of Hypertension, Tongji Hospital, Tongji Medical College of Huazhong University of Science and Technology, Wuhan, (China) Tel. and Fax (86-27)8366-2827 or (86-27)8366-3280, E-Mail dwwang@tjh.tjmu.edu.cn / xzxu@tjh.tjmu.edu.cn 


\section{Cellular Physiology Cell Physiol Biochem 2016;39:721-739 and Biochemistry Published online: July 27, $2016 \quad \begin{aligned} & \text { DOI: 10.1159/000445663 } 2016 \text { The Author(s). Published by S. Karger AG, Basel } \\ & \text { www.karger.com/cpb }\end{aligned}$ Zhou et al.: CYP2J2 Gene Delivery Attenuated Adventitial Remodeling}

\section{Introduction}

Hypertension is a high risk factor of cardiac and cerebrovascular diseases, which is increasing with the aging of population and life style changes. The persistent hemodynamic force of hypertension results in structural alteration of vessel wall [1]. Aorta is composed of three layers: intima, media and adventitia. Unlike intima and media, which are composed of endothelial cells and smooth muscle cells respectively, adventitia layer includes fibroblasts, immunocytes, and adrenergic nerves [2,3]. Previous studies on vascular remodeling have focused on intima and media [4], however, growing evidences suggest that adventitia plays a crucial role in maintaining the homeostasis of vessel wall [5, 6]. Even though abundant researches have been reported, the underlying mechanisms of vascular remodeling are not fully understood. Interestingly, more and more studies support the view that adventitial inflammation contributes to the pathogenesis of vascular remodeling [7].

Cytochrome P450 epoxygenase 2J2 (CYP2J2) is widely expressed in human cardiomyocytes, endothelial cells (ECs) and smooth muscle cells (VSMCs), which metabolizes arachidonic acid to four regioisomeric epoxyeicosatrienoic acids, namely 5,6-, 8,9-, 11,12and 14,15-EET. Soluble epoxide hydrolase (SEH) hydrolyzes EETs to less biologically active dihydroxyeicosatrienoic acids (DHET) [8]. Accumulating evidences suggest that EETs exert important biological activities in various cardiovascular diseases. Overexpression of CYP2J2 prevented the development of hypertension in spontaneous hypertensive rats (SHR) by enhancing atrial natriuretic peptide expression [9]. CYP2J2 gene delivery ameliorated monocrotaline (MCT)-induced pulmonary artery hypertension and pulmonary vascular remodeling by enhancing eNOS activity, inhibiting inflammation and modifying TGF- $\beta$ / BMPR II-smad signaling pathway [10]. Additionally, epoxygenases overexpression or sEH inhibiting offered anti-atherosclerotic effect by inhibiting VSMCs proliferation, migration, and down-regulating inflammatory mediators [11, 12]. Cai et al. [13] reported CYP2J2 overexpression prevented abdominal aortic aneurysm development via PPAR $\gamma$ activation and inflammatory inhibition. These data indicated that CYP/sEH system is involved in the pathogenesis of vascular diseases. However, whether CYP and sEH exist in AFs remains unknown, and whether CYP2J2 gene delivery could attenuate adventitial remodeling needs to be further explored.

Peroxisome proliferator-activated receptor (PPAR) subfamily of nuclear hormone receptors includes three isoforms: PPAR $\alpha, \mathrm{PPAR} \beta / \delta$ and PPAR $\gamma$. PPAR $\gamma$ exerts important roles in cellular differentiation, proliferation, lipid and glucose metabolism [14]. Studies showed that PPAR $\gamma$ ligands suppress inflammation by inhibiting the activation of nuclear factor $\kappa \mathrm{B}(\mathrm{NF}-\kappa \mathrm{B})$, activator protein $1(\mathrm{AP}-1)$ and signal transducers and activator of transcription (STAT). Moshal et al. [15] reported that CYP2J2 transfection or exogenous 8,9EET addition phosphorylated AKT and attenuated homocysteine-induced MMP-9 activation by inhibiting NF- $\kappa$ B nuclear translocation, NF- $\kappa B-D N A$ binding, and I $\kappa \mathrm{B} \alpha$ activation. Further studies indicated that PPAR $\gamma$ is an effector of EETs, as blocking PPAR $\gamma$ by GW9662 abolished EET/AUDA-mediated anti-inflammatory effect [16]. These results concluded that the antiinflammatory effect of EETs is partially mediated by PPAR $\gamma / \mathrm{NF}-\kappa \mathrm{B}$ signaling pathway. Janus kinase (JAK)/STAT cascade is well known to be involved in the inflammatory response, and suppressor of cytokine signaling (SOCS) negatively regulates JAK/STAT cascade by a feedback mechanism. PPAR $\gamma$ ligands, $15 \mathrm{~d}-\mathrm{PG} 22$ and rosiglitazone rapidly induced the transcription of SOCS1 and SOCS3, which in turn to inhibit JAK activity in activated glial cells [17]. Moreover, 15d-PGJ2 and troglitazone suppressed pancreatitis by inhibiting JAK2/STAT3 pathway through SOCS3 induction [18]. Hence, EETs, as activator of PPAR $\gamma$, have potential to regulate JAK/STAT signaling pathway.

In this study, firstly, we explore whether CYP/sEH system exists in human aortic adventitia, and whether participates in adventitial remodeling process. Then we used C57BL/6J mice in vivo and primary rat adventitial fibroblasts in vitro treated with Ang II to investigate whether CYP2J2 gene delivery and exogenous EETs administration could prevent Ang II induced adventitial remodeling. 


\section{Cellular Physiology Cell Physiol Biochem 2016;39:721-739

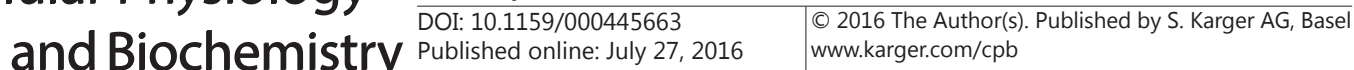 Zhou et al.: CYP2J2 Gene Delivery Attenuated Adventitial Remodeling}

\section{Materials and Methods}

\section{Human aortic adventitia samples}

The design of this study complied with the Declaration of Helsinki, and was approved by the Review Board of Tongji Hospital, Tongji Medical College, Huazhong University of Science and Technology. The subjects recruited to this study provided written informed consents. We obtained aortas from five patients undergoing aortic replacement surgery due to aortic dissection. Patients with a history of myocardial infarction and arteritis were excluded. Normal aortic vessels were obtained from age-matched traffic accident victims. The detailed method for human aortic adventitia isolation from media and intima was described previously [19].Tissue samples were obtained and kept frozen in liquid nitrogen, then stored at $-80^{\circ} \mathrm{C}$ before use.

\section{Primary adventitial fibroblasts cell culture}

Adventitial fibroblasts were isolated from normal thoracic aortas of 8 week-old male Wistar-Kyoto rats as described previously [20] with some modification. Briefly, rats were anesthetized, parietal pleura were opened, and the full length of thoracic aortas were removed into cold PBS rapidly. Under sterilization, loose connective tissue was separated and segmental arteries were cut off slightly. Then aortic lumen was opened, the endothelium was rubbed and the media was distinguished gently using forceps. The remaining adventitia was minced carefully into small pieces $\left(1-2 \mathrm{~mm}^{3}\right)$ in FBS, placed on $0.1 \%$ gelatin-coated dishes and cultured with high glucose DMEM containing 10\% FBS, 100U/mL penicillin and streptomycin. The explants were incubated in a humidified incubator at $37^{\circ} \mathrm{C}$ with $5 \% \mathrm{CO}_{2}$, and the medium was changed every 3 days. 3-5 days later, AFs grew out from tissues and reached confluence after 7-10 days (Fig. 2A). The purity of AFs was identified by immunofluorescence of Vimentin (AFs marker) and $\alpha$-SMA (VSMCs marker). Experiments were performed on passages 3-6.

\section{Immunofluorescence}

Cells were fixed in $4 \%$ paraformaldehyde for $30 \mathrm{~min}$, then permeated by $0.2 \%$ Triton X-100 for 20 min and blocked with $5 \%$ bovine serum albumin for $2 \mathrm{~h}$ at room temperature. The primary antibodies against Vimentin and $\alpha$-SMA at concentration of $1: 100$ were used at $4{ }^{\circ} \mathrm{C}$ overnight, followed by incubation with Cy3 or FITC-labelled secondary antibody (1:100) for $2 \mathrm{~h}$ at room temperature avoid from light. Nuclei was stained with DAPI, then washed with TBST for $5 \mathrm{~min}, 3$ times. Samples were examined by fluorescence microscope.

\section{SOCS3 siRNA transfection}

50 nM SOCS3 siRNA (Ruibo, Guangzhou, China) was transfected into AFs with Lipofectamine 2000 reagent (Invitrogen, CA) according to the manufacturer`s instructions. Transfected efficiency was determined by Western blot $36 \mathrm{~h}$ after transfection. Experiments were subsequently performed only if the SOCS3 silent effect was more than $70 \%$.

\section{AFs proliferation assay}

CCK-8 (Dojindo, Japan) assay was used to measure cell proliferation. AFs were seeded into a 96-well plate with $2 \times 10^{3}$ cells/well in $100 \mu \mathrm{l}$. After $24 \mathrm{~h}$ incubation, cells were pretreated with GW9662 $(1 \mu \mathrm{M})$ or transfected SOCS3 siRNA, then 11,12-EET $(1 \mu \mathrm{M})$ was added, followed by Ang II $\left(10^{-7} \mathrm{M}\right)$ stimulation for 24 $\mathrm{h}$. At the end of intervention, $10 \mu \mathrm{l}$ CCK-8 solution was added to each well of the plate, and incubated for $2 \mathrm{~h}$. The absorbance at $450 \mathrm{~nm}$ was measured using a microplate reader. The relative cell viability was calculated as $\left(\mathrm{OD}_{450}\right.$ of treated samples- $\mathrm{OD}_{450}$ of control samples) / $\left(\mathrm{OD}_{450}\right.$ of untreated samples- $\mathrm{OD}_{450}$ of control samples) $\times 100 \%$. Each experiment group had 5 duplicate wells and repeated 4 times.

\section{AFs migration assay}

Transwell cell culture chamber (Corning, New York, USA) was used to allow cells to migrate through $8.0 \mu \mathrm{m}$ pore size polycarbonate membrane to observe AFs migration in vitro [21]. Briefly, $3 \times 10^{4}$ cells in 100 $\mu \mathrm{l}$ serum free DMEM were seeded into the upper chambers, while $600 \mu \mathrm{l}$ serum free DMEM supplemented with different stimulus were performed in the lower chamber. Incubation was continued at $37^{\circ} \mathrm{C}$ with $5 \% \mathrm{CO}_{2}$ for $6 \mathrm{~h}$, after which the filters were removed. Cells remaining on the upper surface of membrane 


\section{Cellular Physiology Cell Physiol Biochem 2016;39:721-739 \begin{tabular}{c|c|c|} 
DOI: 10.1159/000445663 & $\begin{array}{l}\text { O 2016 The Author(s). Published by S. Karger AG, Basel } \\
\text { wwww.karger.com/cpb }\end{array}$
\end{tabular} \\ Zhou et al.: CYP2J2 Gene Delivery Attenuated Adventitial Remodeling}

(not migrated) were gently rubbed off with a moist cotton swab, and the migrated cells attached on the lower surface were fixed with methanol for $20 \mathrm{~min}$, and then stained with $0.2 \%$ crystal violet for $1 \mathrm{~h}$. The number of migrated cells was quantified by counting 5 random fields $(\times 100)$ in each membrane using a light microscope.

\section{Western blot analysis}

Following stimulation, cells and aortas were extracted and homogenized, nuclear protein was extracted using Boster Kit according to the manufacturer's instructions [22]. The protein concentration was measured using BCA protein assay Kit. Totally $40 \mu \mathrm{g}$ protein was subjected to $10 \%$ SDS-PAGE gel for separation, and then transferred into PVDF membrane. After blocking with $5 \%$ non-fat milk for $2 \mathrm{~h}$, the membranes were incubated with primary antibodies against $\alpha$-SMA, Collagen I, PPAR $\gamma, \operatorname{PPAR} \alpha, \operatorname{I\kappa B} \alpha, \mathrm{p}-\mathrm{I} \kappa \mathrm{B} \alpha, \mathrm{NF}-\kappa \mathrm{B}$ p 65, Lamin B1, CYP2J2, sEH, $\beta$-actin (Santa Cruz Biotechnologies), SOCS3, p-JAK2, JAK2, p-STAT3 and STAT3 (Cell Signaling Technology) overnight at $4^{\circ} \mathrm{C}$. After washing by TBST, the membranes were incubated with horseradish peroxidase-conjugated antibody for $2 \mathrm{~h}$ at room temperature. Bands were visualized using ECL and quantified with Gel Pro analysis software. $\beta$-actin and Lamin B1 were used as reference for total or cytosolic protein and nuclear protein, respectively.

\section{EMSA}

Nuclear extracts were prepared as instructions indicated [23]. Oligonucleotides containing NF- $\mathrm{kB}$ binding site (5'-AGTTGAGGGGACTTTCCCAGGC-3') end-labeled with biotin was obtained from Invitrogen. Briefly, The biotin-labeled probe was incubated with $10 \mu \mathrm{g}$ nuclear extracts for $30 \mathrm{~min}$ at room temperature in $50 \mathrm{mM}$ Tris- $\mathrm{HCl}$ buffer (pH 7.5) containing 2.5\% glycerol, $5 \mathrm{mM} \mathrm{MgCl}, 50 \mathrm{mM} \mathrm{KCl}, 10 \mathrm{mM}$ EDTA, $0.05 \%$ $\mathrm{NP}-40$ and $0.05 \mu \mathrm{g} / \mu \mathrm{l}$ poly (dl.dc). The protein-DNA complexes were separated in a $5 \%$ nondenaturing PAGE gel. The relative bands intensity was analyzed using Gel pro-analysis software.

\section{RNA extraction and quantitative RT-PCR}

Total RNA was extracted and reversely transcribed using TRIzol (Invitrogen) and MultiScribe (ABI). Real-time PCR analysis was performed on a 7900HT Fast Real-Time PCR System (ABI). The relative amounts of specific gene transcripts were calculated according to the comparative threshold cycle $\left(2^{-\Delta \Delta \mathrm{Ct}}\right)$ method. Primer sequences for quantitative real-time RT-PCR were shown as following: SOCS3 forward: $5^{\circ}$-CCG AAG CAC GCA GCC AG-3`; reverse: 5`TCT CGC CCC CAG AAT AGA TGT A-3`; $\beta$-actin forward: 5 `-GCT CTA TCC TGG CCT CAC TGT C-3`; reverse: 5`-TTG TCA AAG AAA GGG TGT AAA ACG-3`.

\section{rAAV vectors preparation}

We used HEK 293T cells to produce type 2 rAAV vectors containing CYP2J2 or green fluorescent protein (GFP) by triple plasmid co-transfection as described previously [24]. The vectors were purified, diluted and stored at $-80^{\circ} \mathrm{C}$ before injection.

\section{Gene delivery and Animals models}

All procedures were approved by the Institutional Animal Care and Use Committee of Tongji Medical College, and conducted in conformity with institutional guidelines. 8 week-old C57BL/6J male mice were purchased from SLAC Laboratory Animal Co, Ltd (Shanghai, China). Animals were treated before experiment. $100 \mu \mathrm{lAAV}$ vectors $\left(1 \times 10^{11} \mathrm{pfu}\right)$ were injected via tail veins 2 weeks before Ang II infusion. Adventitial remodeling model was induced by a 14 days continuous Ang II $(800 \mu \mathrm{g} / \mathrm{kg} / \mathrm{d})$ infusion by subcutaneous osmotic pumps (Alzet, Model 1002, USA) implantation [25]. Mice were randomly divided into 5 groups: GFP group, receiving rAAV-GFP injection; 2J2 group, receiving rAAV-2J2 injection; Ang II + GFP group, receiving Ang II infusion with rAAV-GFP injection; Ang II + 2J2 group, receiving Ang II infusion with rAAV-2J2 injection; Ang II + Hydralazine group, receiving Ang II infusion with hydralazine orally ( $250 \mathrm{mg} / \mathrm{L}$ in drinking water) [26]. Each group included 6 mice.

\section{Blood pressure measurement}

Tail cuff method was used to measure systolic blood pressure (SBP) every week with a blood pressure monitor (BP-2010A, Softron, Japan). All mice were first trained daily for 3 days before this study. SBP was determined averaged of 6 consecutive measurements. 


\section{Cellular Physiology Cell Physiol Biochem 2016;39:721-739

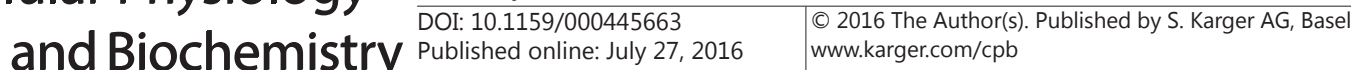 \\ Zhou et al.: CYP2J2 Gene Delivery Attenuated Adventitial Remodeling}

Histological analysis

Mice were sacrificed at the end of the intervention. We obtained the mouse aortas as previous reported [27]. This method has no effect on the aortic adventitial integrity and thickness measurements. Thoracic aortas of each group were harvested, fixed in 4\% paraformaldehyde, and embedded in paraffin for histological analysis. Sections were stained with hematoxylin and eosin, and sirius red. Immunohistochemical staining of CYP2J2, $\alpha$-SMA and PCNA were performed according to the manufacturer's instructions (Zsbio, China).

Evaluation of vascular and circulating EETs levels

EET levels in vessel and serum were analyzed by using both LC/MS and ELISA kits (Detroit R\&D, USA) as described previously [28, 29].

ELISA kits for inflammatory cytokines (IL-6, MCP-1)

The levels of IL- 6 and MCP-1 in culture medium and mouse serum were assayed by using ELISA kits from R\&D systems following the manufacturer`s instructions.

Statistical analysis

Continuous data were expressed as means \pm S.E.M. All data analysis was performed with SPSS 17.0 statistical software. Comparisons between groups were performed by one-way analysis of ANOVA with post hoc analyses performed using the Student-Newman-Keuls method. Two-way ANOVA analysis was used in Comparisons of mouse SBP value between different treatment groups and time points. $p<0.05$ was considered statistically significant.

\section{Results}

CYP 2J2 and sEH expression in human aortic adventitia

To investigate the potential role of $\mathrm{CYP} / \mathrm{sEH}$ system on adventitial remodeling, CYP2J2 and sEH expressions were detected in human aortic adventitia. Results showed that CYP2J2 expression was lower in adventitia than intima and media, and the levels of sEH in media and adventitia were higher than intima (Fig. 1A). Interestingly, upregulation of sEH and downregulation of CYP2J2 expression were observed in adventitia of patients with aortic dissection, compared with that in normal population (Fig. 1B, Fig. 1C), which probably resulted in a net decrease in EETs level in adventitia. Taken together, these data demonstrated that CYP/sEH system existed in adventitia, and was involved in adventitial remodeling process.

\section{EETs inhibit Ang II-induced AFs differentiation}

Adventitial remodeling is characterized by adventitia thickening, AFs proliferation, migration, phenotypic transformation, and extracellular matrix deposition [30]. Firstly, the cultured cells were stained negative for $\alpha$-SMA, but positive for Vimentin, meanwhile both Vimentin and $\alpha$-SMA were positive in VSMCs (Fig. 2A). Our data indicated that AFs were successfully isolated with a high purity.

Ang II is a potent inducer that stimulates phenotypic transformation of AFs into myofibroblasts, which is marked by $\alpha$-SMA upregulation [31]. To explore the potential role of EETs on Ang II-induced AFs differentiation in vitro, $\alpha$-SMA expression was detected by Western blot and immunofluorescence staining. As expected, the basic $\alpha$-SMA expression in AFs was very low, and 11,12-EET, GW9662, SOCS3 siRNA had no impact on $\alpha$-SMA expression. However, the expression of $\alpha$-SMA was significantly increased in Ang II group, while pretreatment with 8,9-EET, 11,12-EET, 14,15-EET remarkably alleviated Ang IIinduced AFs differentiation, in which 11,12-EET exerted the highest efficiency $(p<0.05)$ (Fig. 2B). PPAR $\gamma$ antagonist GW9662 incubation and SOCS3 siRNA transfection before 11,12-EET treatment partially reversed the protective effect of 11,12-EET ( $p<0.05)$ (Fig. 2C, Fig. 2D). In addition, 11,12-EET decreased AFs transformation to MFs determined by immunofluorescence staining, while GW9662 and SOCS3 siRNA alleviated these effects (Fig. 


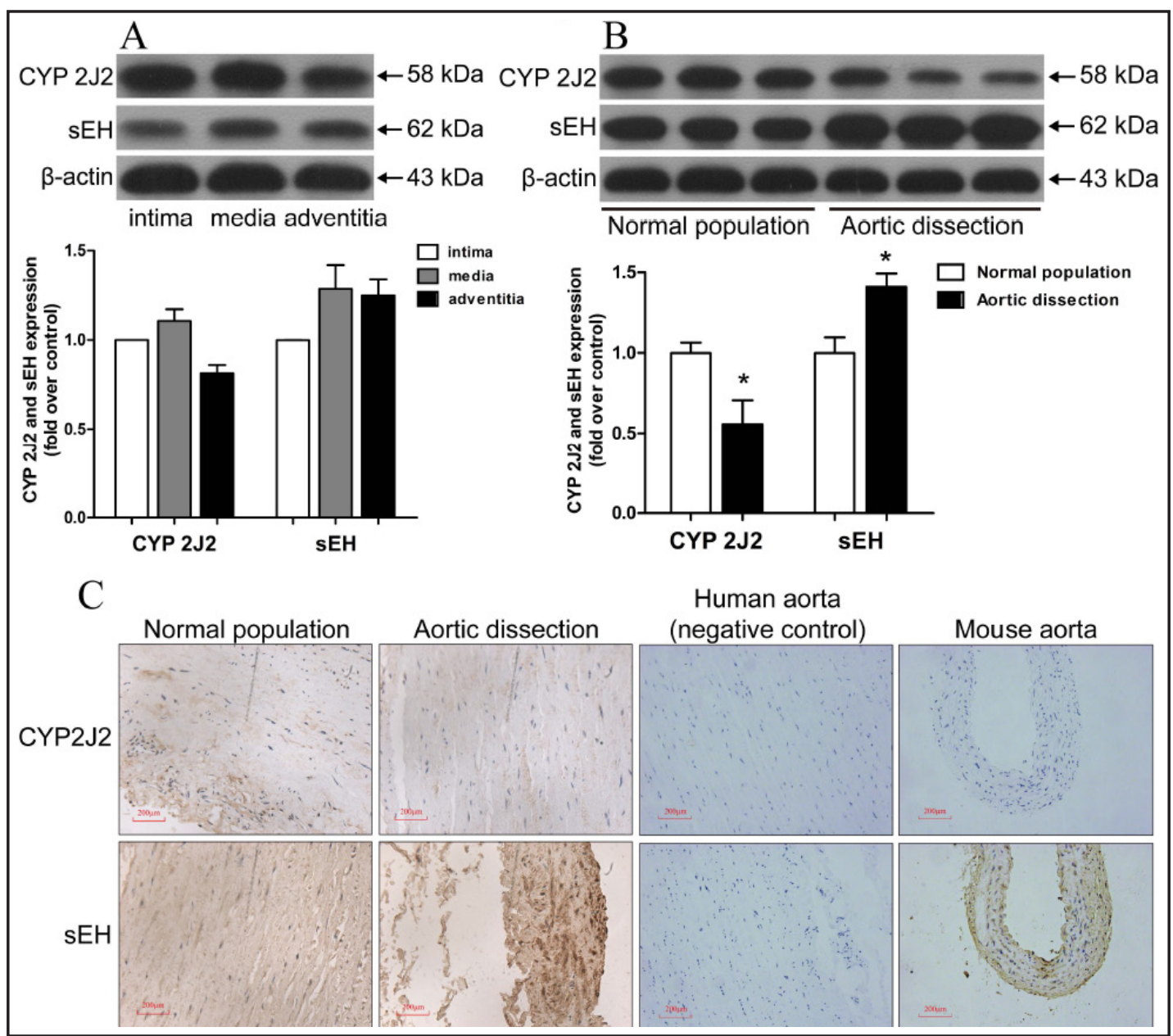

Fig. 1. CYP2J2 and sEH express in human aortic adventitia. (A) CYP2J2 and sEH expressed in different layers of human aorta. (B) SEH was activated and CYP2J2 was inhibited in adventitia of aortic dissection patients. (C) CYP2J2 and sEH expression in human aortic adventitia from normal population and aortic dissection patients. ${ }^{*} p<0.05$ vs. normal population. Data were expressed as mean $\pm \mathrm{SEM}, \mathrm{n}=3$ for each group.

2E). These data indicated that exogenous EETs inhibited Ang II-induced AFs differentiation via PPAR $\gamma$ and SOCS3 signaling pathways.

\section{EETs inhibit Ang II-induced AFs proliferation}

To evaluate the effect of EETs on Ang II accelerated AFs proliferation, AFs were pretreated with 11,12-EET $0.5 \mathrm{~h}$ before Ang II stimulation for $24 \mathrm{~h}$. Compared with vehicle group, the $\mathrm{OD}_{450}$ ratio in Ang II group increased by $46 \%$. Interestingly, a significant antiproliferative effect was observed in Ang II+11,12-EET group (15.72\%) $(p<0.05)$ (Fig. 2F). When GW9662 $(1 \mu \mathrm{M})$ was added $0.5 \mathrm{~h}$ or SOCS3 siRNA was transfected $36 \mathrm{~h}$ before 11,12EET administration, the effect of 11,12-EET was reversed ( $p<0.05)$ (Fig. 2F, Fig. 2G).

\section{EETs inhibit Ang II-induced AFs migration}

As shown in Fig. 3A, Ang II $\left(10^{-7} \mathrm{M}\right)$ stimulation promoted AFs migration by 7.08 folds compared with control group. Interestingly, 11,12-EET pretreatment significantly reduced these chemotactic effects to 2.81 folds $(p<0.05)$. We next examined the pathway involved in the anti-migratory effect. Results showed that GW9662 pretreatment partially blocked the beneficial effect of 11,12-EET on AFs migration $(p<0.05)$ (Fig. 3A). Additionally, silencing SOCS3 expression by siRNA also reversed the anti-migratory property of 11,12 -EET $(p<0.05)$ 


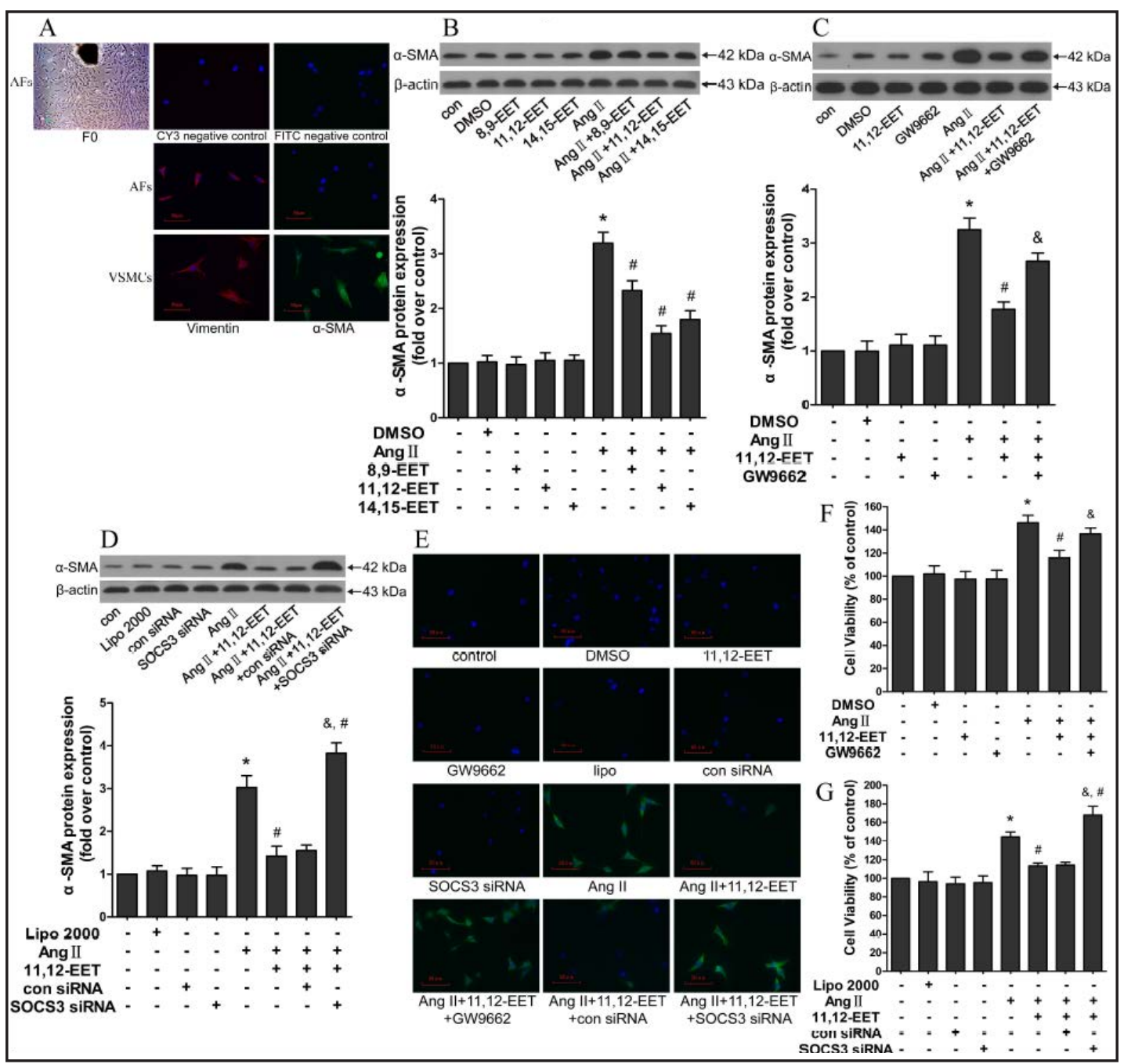

Fig. 2. Effect of exogenous EET treatment on Ang II-induced AFs differentiation and proliferation. (A) Immunofluorescent staining of Vimentin and $\alpha$-SMA in cultured AFs and VSMCs (Red: Cy3, Green: FITC, blue: DAPI). (B) 8,9-, 11,12-, 14,15-EET attenuated Ang II-induced AFs differentiation. (C) The protective effect of 11,12-EET on AFs differentiation was reversed by PPAR $\gamma$ antagonist GW9662. (D) The protective effect of 11,12-EET on AFs differentiation was reversed by SOCS3 siRNA transfection. (E) Immunofluorescent staining of AFs transformation to MFs (Green: FIFC, blue: DAPI). (F) GW9662 pretreatment alleviated 11,12EET effect on AFs proliferation. (G) SOCS3 siRNA transfection alleviated 11,12-EET effect on AFs proliferation. Data were expressed as mean \pm SEM, $\mathrm{n}=4$ for each group, ${ }^{*} p<0.05$ vs. control, $\# p<0.05$ vs. Ang II, $\& p<0.05$ vs. Ang II+11,12-EET.

(Fig. 3B). These data suggested that the inhibitory effect of EETs on Ang II-induced AFs migration was involved in PPAR $\gamma$ activation and SOCS3 induction.

EETs inhibit Ang II-induced AFs collagen synthesis

Ang II emerges as a major profibrogenic cytokine of tubulointerstitial fibrosis [32]. As expected, Ang II $\left(10^{-7} \mathrm{M}\right)$ incubation enhanced collagen I synthesis in AFs, and 11,12-EET pretreatment attenuated these effects of Ang II $(p<0.05)$ (Fig. 3C). However, 11,12-EET alone had no effect on basal collagen I expression. Interestingly, these effects of 11,12-EET were reversed by GW9662 incubation and SOCS3 siRNA transfection $(p<0.05)$ (Fig. 3C, Fig. 3D). These data indicated that 11,12-EET inhibited Ang II-induced AFs collagen synthesis via PPAR $\gamma$ and SOCS3 dependent manner. 


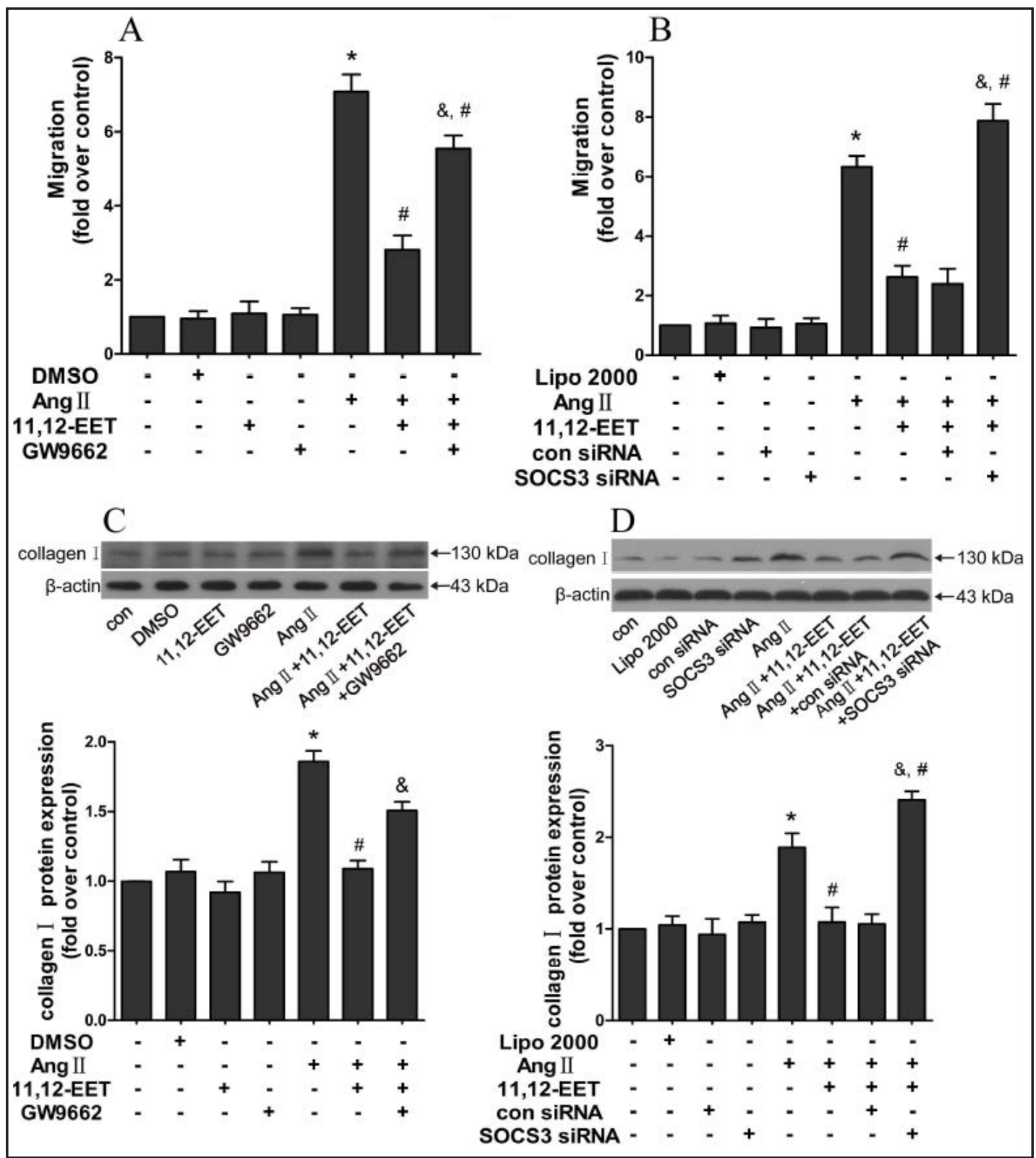

Fig. 3. Effect of exogenous EET treatment on Ang II-induced AFs migration and collagen synthesis. (A) PPAR $\gamma$ antagonist GW9662 pretreatment reversed 11,12-EET effect on AFs migration. (B) SOCS3 siRNA transfection reversed 11,12-EET effect on AFs migration. (C) 11,12-EET reduced Ang II-induced collagen I expression in AFs, while GW9662 reversed this effect. (D) SOCS3 siRNA transfection alleviated 11,12-EET effect on collagen I expression induced by Ang II in AFs. Data were expressed as mean \pm SEM, $n=4$ for each group, ${ }^{*} p<0.05$ vs. control, $\# p<0.05$ vs. Ang II, $\& p<0.05$ vs. Ang II+11,12-EET.

The anti-inflammatory effect of EETs is mediated by PPAR $\gamma$-dependent $N F-\kappa B$ pathway

PPAR $\gamma$ is an effector of EETs, which is involved in EET/AUDA-mediated antiinflammatory effect [16]. As shown in Fig. 4A, 11,12-EET remarkably induced PPAR $\gamma$ protein expression in AFs, while Ang II reduced PPAR $\gamma$ expression, which was reversed by 11,12EET incubation. Interestingly, we also found exogenous 11,12-EET remarkably activated PPAR $\alpha$, and partially reversed Ang II induced PPAR $\alpha$ downregulation in AFs (Fig. 5A). These results were in consistent with that reported by Wray JA [33]. However, PPAR $\alpha$ antagonist GW6471 did not abolish the effect of 11,12-EET on Ang II-induced AFs differentiation 


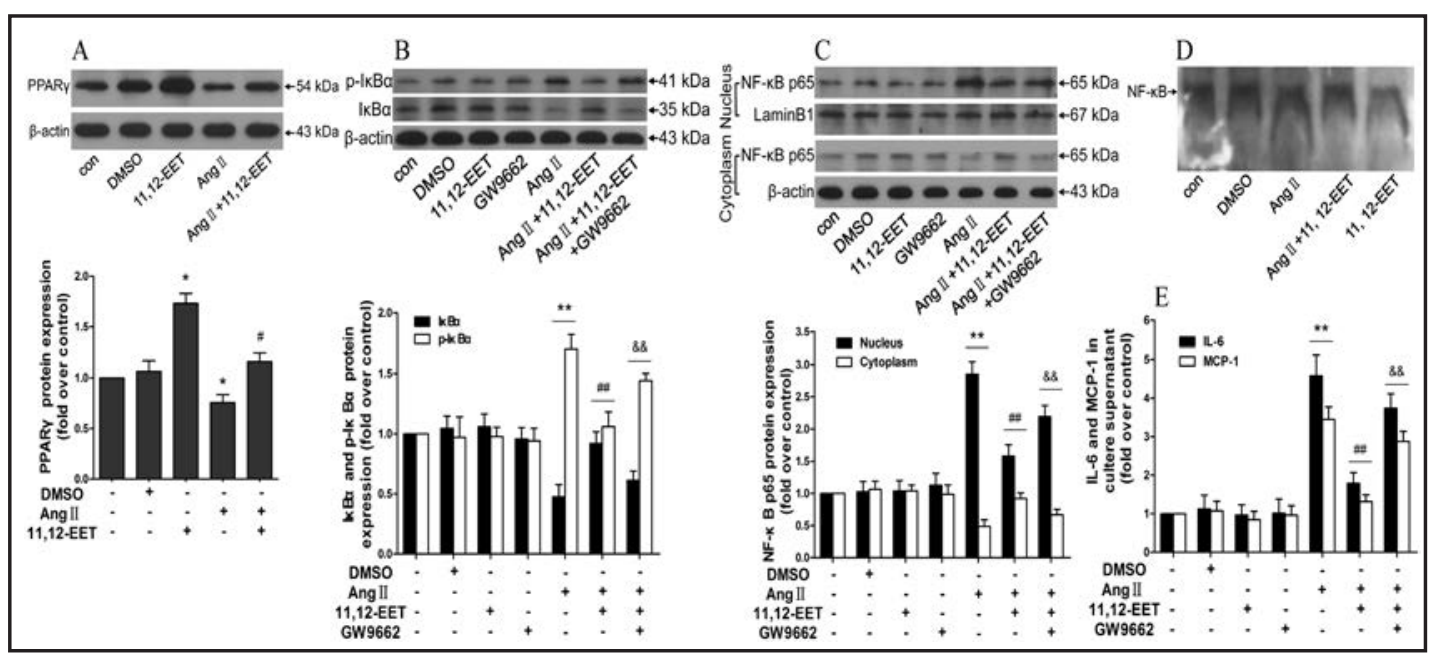

Fig. 4. The effect of 11,12-EET on Ang II-induced inflammatory response was mediated by PPAR $\gamma$-NF- $\mathrm{B}$ signaling pathway in AFs. (A) 11,12-EET increased PPAR $\gamma$ expression, and reversed Ang II-induced PPAR $\gamma$ reduction. (B) 11,12-EET reduced Ang II-induced I $\kappa \mathrm{B} \alpha$ phosphorylation, and GW9662 blocked this effect. (C) 11,12-EET reduced Ang II-induced NF- $\mathrm{kB}$ nuclear translocation, and GW9662 reversed this effect. (D) Ang II-induced NF- $\kappa \mathrm{B}$ transcriptive activity was abolished by 11,12-EET incubation. (E) 11,12-EET pretreatment reduced Ang II-induced inflammatory cytokines IL-6 and MCP-1 secretion, and GW9662 reversed these effects. Data were expressed as mean \pm SEM, $\mathrm{n}=4$ for each group, ${ }^{*} p<0.05$ vs. control, $\# p<0.05$ vs. Ang II, $\& p<0.05$ vs. Ang II + 11,12-EET.

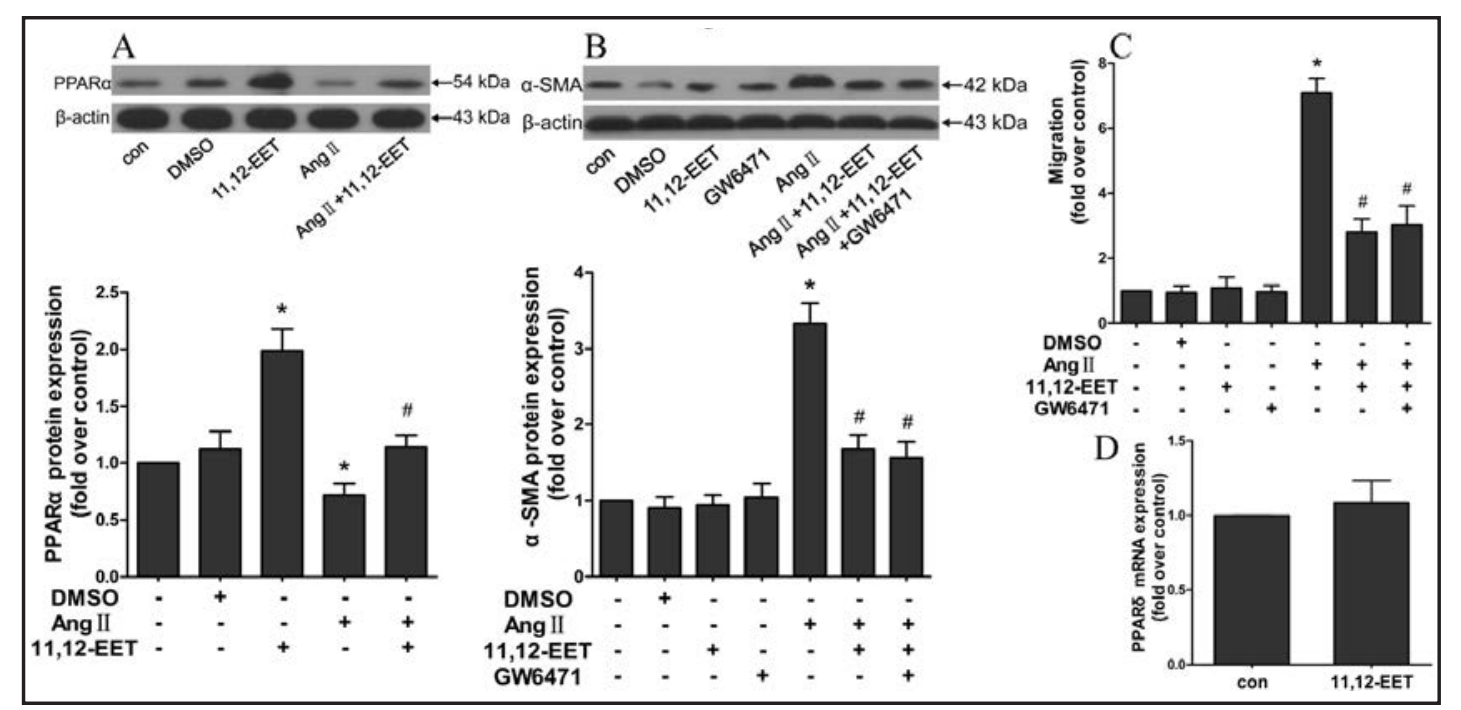

Fig. 5. The effect of 11,12-EET on PPAR $\alpha$ and PPAR $\delta$ in adventitial remodeling. (A) 11,12-EET increased PPAR $\alpha$ expression, and reversed Ang II-induced PPAR $\alpha$ reduction in AFs. (B) The protective effect of 11,12EET on AFs differentiation was not reversed by PPAR $\alpha$ antagonist GW6471. (C) GW6471 pretreament did not reversed 11,12-EET effect on AFs migration. (D) 11,12-EET exerted no effect on PPAR $\delta$ mRNA expression. Data were expressed as mean \pm SEM, $\mathrm{n}=4$ for each group, ${ }^{*} p<0.05$ vs. control, $\# p<0.05$ vs. Ang II, $\& p<0.05$ vs. Ang II + 11,12-EET.

and migration as shown in Fig. 5B-5C. Moreover, EETs exerted no influence on the PPAR $\delta$ mRNA expression (Fig. 5D). Ang II incubation potentiated I $\kappa$ B $\alpha$ phosphorylation (Fig. 4B), which led to a significant elevation of NF- $\kappa B$ nuclear translocation (Fig. 4C). Interestingly, 11,12-EET pretreatment remarkably attenuated Ang II-induced I $\kappa \mathrm{B} \alpha$ phosphorylation and subsequent NF- $\kappa$ B nuclear translocation. These effects of 11,12-EET were partially abolished KARGER 


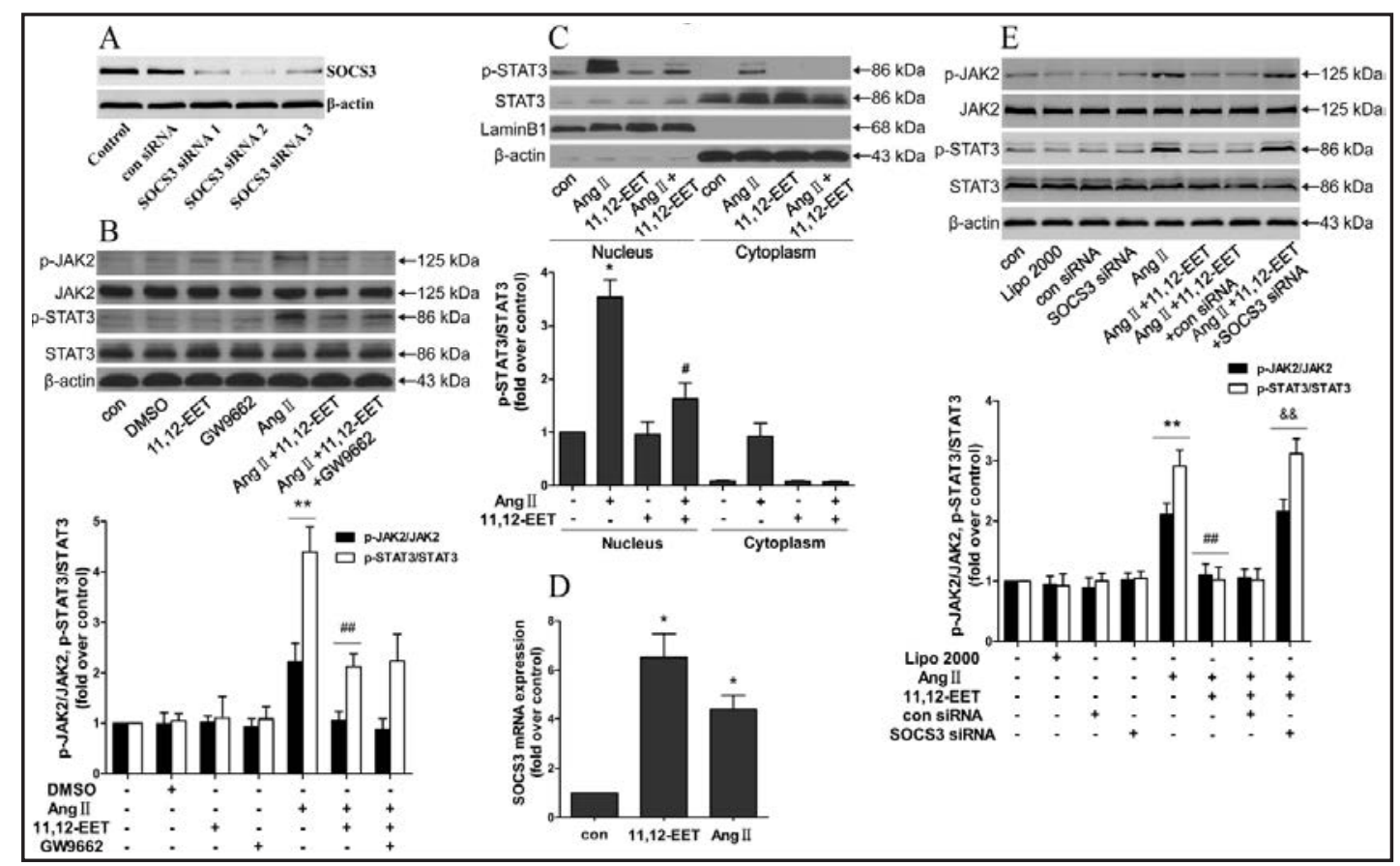

Fig. 6. The effects of 11,12-EET on Ang II-induced inflammatory response was mediated by SOCS3/JAK/ STAT pathway in AFs. (A) SOCS3 expression declined after SOCS3 siRNA were transfected into AFs. (B) Ang II-induced JAK2, STAT3 phosphorylation in AFs was reduced by 11,12-EET incubation, but GW9662 did not reversed these effects. (C) 11,12-EET reduced Ang II-induced phosphor-STAT3 nuclear translocation. (D) 11,12-EET incubation and Ang II stimulation increased SOCS3 mRNA expression in AFs. (E) SOCS3 knockdown in AFs prevented the inhibitory effects of EETs on Ang II-induced JAK/STAT activation. Data were expressed as mean \pm SEM, $n=4$ for each group, ${ }^{*} p<0.05$ vs. control, $\# p<0.05$ vs. Ang II, $\& p<0.05$ vs. Ang II + 11,12-EET.

by PPAR $\gamma$ antagonist GW9662 administration ( $p<0.05$ ) (Fig. 4B-4C). Furthermore, 11,12EET pretreatment blocked Ang II-induced activation of transcription factor NF- $\kappa \mathrm{B}$, while 11,12-EET alone had no effect on the basal level (Fig. 4D). We also showed that 11,12-EET pretreatment attenuated Ang II-induced inflammatory cytokine IL- 6 and MCP- 1 secretion in AFs, while GW9662 pretreatment reversed these effects (Fig. 4E). These data suggested that 11,12-EET inhibited Ang II-induced AFs inflammatory response through PPAR $\gamma$-dependent NF- $\kappa$ B signaling pathway.

PPARY independent SOCS3/JAK/STAT pathway is involved in the beneficial effects of EETS on adventitial remodeling

To further explore the potential mechanisms of EETs in AFs activation, we tested whether the JAK/STAT signaling cascade was involved in this event. As shown in Fig. 6A, the expression of SOCS3 significantly declined when $50 \mathrm{nM}$ different kinds of SOCS3 siRNA were transfected into AFs, and the second one exerted the highest silent effect. We selected this SOCS3 siRNA sequence in following experiment. Excitingly, Ang II stimulation induced JAK2 and STAT3 phosphorylation in AFs, and these effects were abolished by 11,12-EET administration (Fig. 6B). Interestingly, PPAR $\gamma$ antagonist GW9662 pretreatment did not affect this effect of 11,12EET, which indicated that these effects of 11,12-EET was independent on PPAR $\gamma$ activation. Furthermore, the level of phosphor-STAT3 in nuclear fraction was found to increase in Ang II group, while the majority of total STAT-3 was present in cytoplasm (Fig. 6C). Importantly, 11,12-EET incubation reduced the nuclear translocation of phosphor-STAT3 (Fig. 6C). SOCS3 was a negative feedback regulator of JAK/STAT pathway [34]. We found that 11,12-EET rapidly upregulated SOCS3 mRNA level in AFs, which was cooperated with Ang II stimulation 


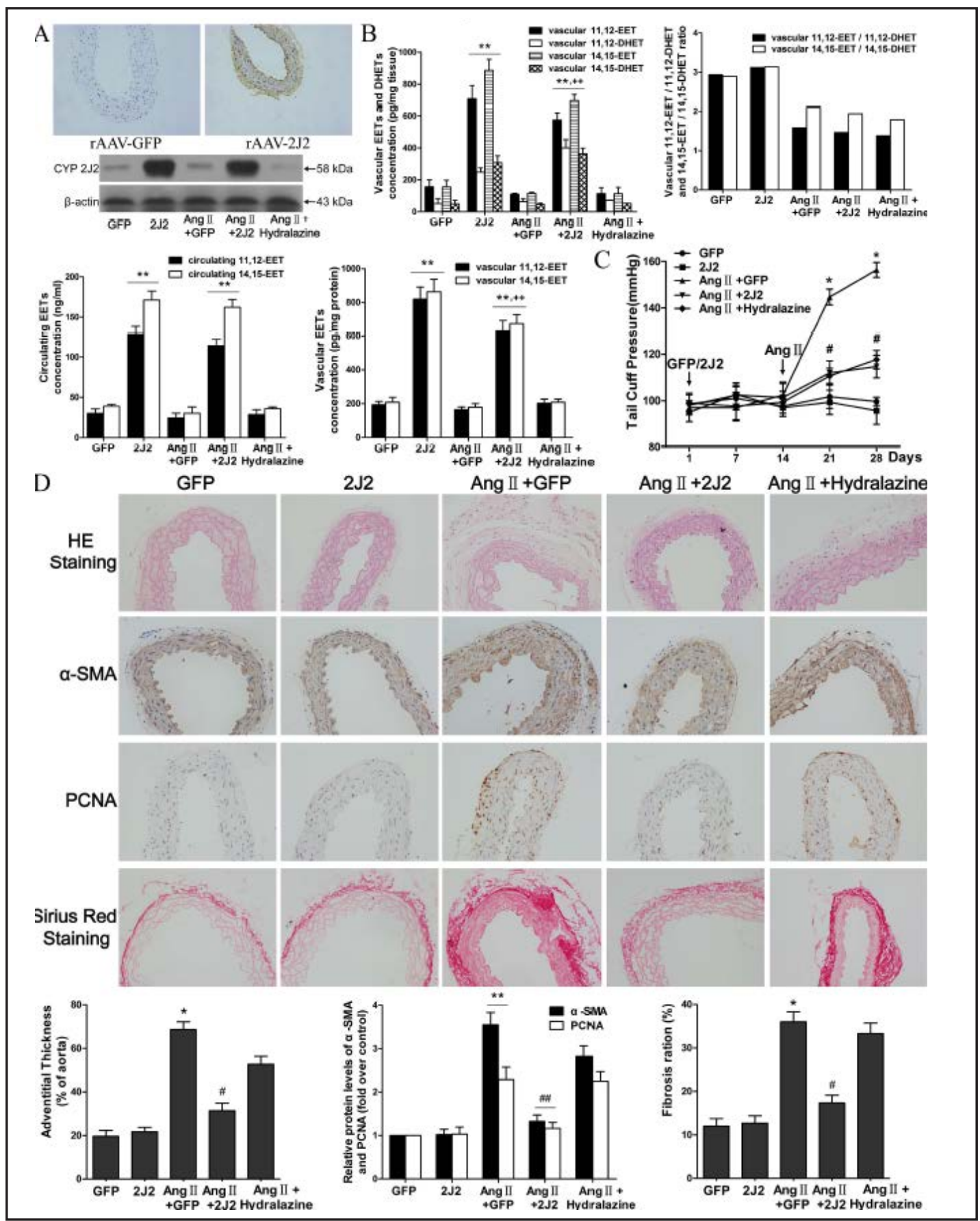

Fig. 7. CYP2J2 gene delivery attenuated Ang II-induced adventitial remodeling in C57BL/6J mice. (A) CYP2J2 was overexpressed in aortic adventitia after rAAV-CYP2J2 injection. (B) CYP2J2 gene delivery increased vascular and circulating EETs and DHETs levels. (C) CYP2J2 overexpression reduced Ang II-induced SBP increasement. (D) CYP2J2 overexpression reduced vessel wall thickening, AFs differentiation, proliferation and collagen deposition induced by Ang II infusion. Data were expressed as mean \pm SEM, $n=6$ for each group, ${ }^{*} p<0.05$ vs. GFP, $\# p<0.05$ vs. Ang II + GFP, $+p<0.05$ vs. CYP2J2.

(Fig. 6D). Additionally, SOCS3 knockdown in AFs by SOCS3 siRNA administration prevented the inhibitory effects of EETs on Ang II-induced JAK/STAT activation (Fig. 6E), which was in consistent with previous study [35]. These results suggested that 11,12-EET inhibited

\section{KARGER}




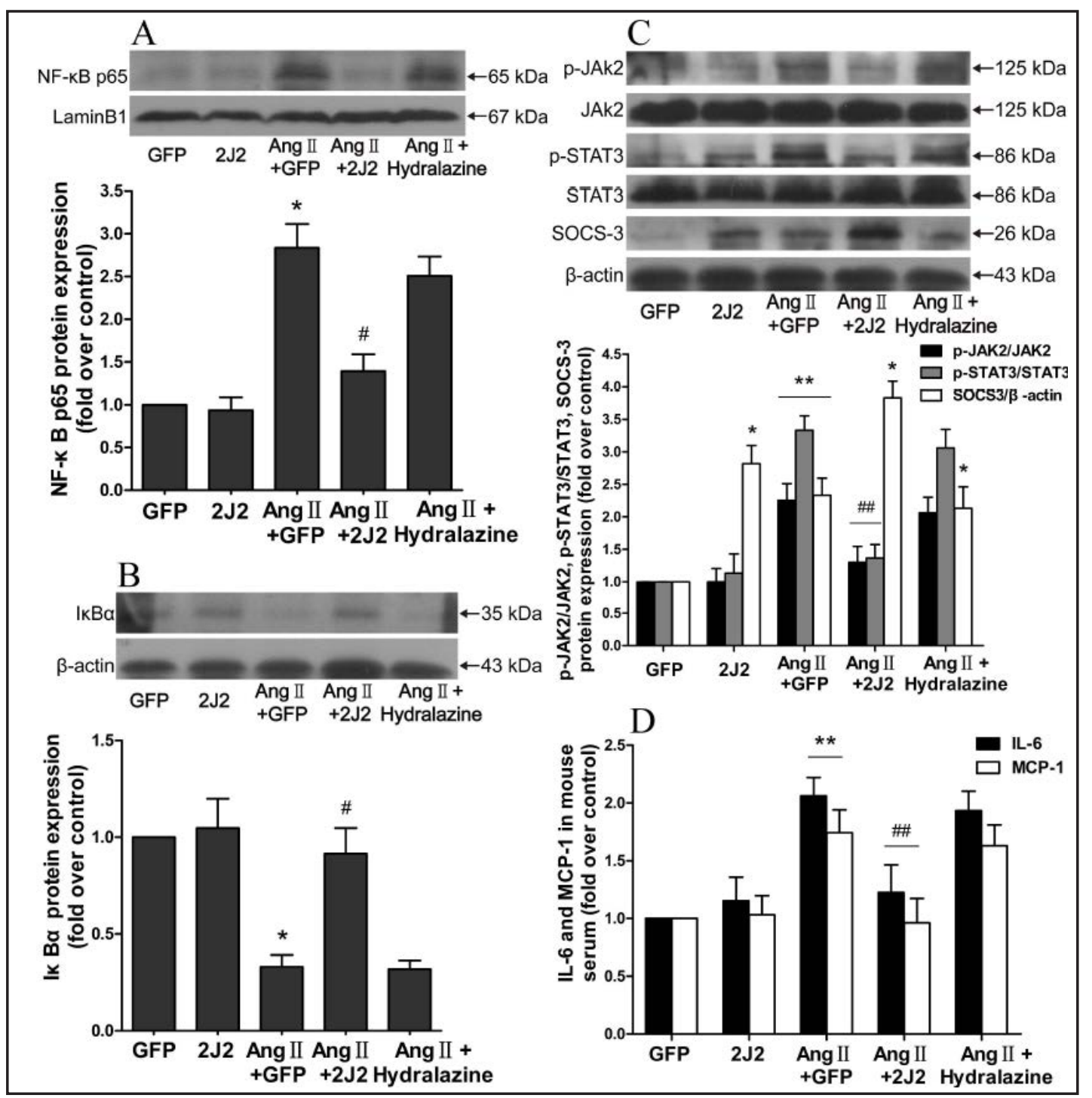

Fig. 8. CYP2J2 gene delivery regulated PPAR $\gamma-\mathrm{NF}-\kappa \mathrm{B}$ and SOCS3/JAK/STAT pathways in adventitial remode-

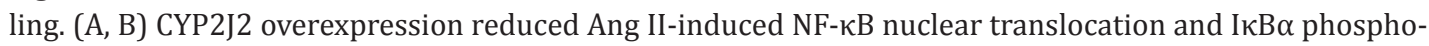
rylation in aorta. (C) CYP2J2 overexpression reduced Ang II-induced JAK2, STAT3 phosphorylation in aorta. (D) CYP2J2 overexpression suppressed Ang II-induced inflammatory cytokines IL-6 and MCP-1 secretion. Data were expressed as mean $\pm \mathrm{SEM}, \mathrm{n}=6$ for each group, ${ }^{*} p<0.05$ vs. GFP, $\# p<0.05$ vs. Ang II $+\mathrm{GFP},+p<0.05$ vs. CYP2J2.

Ang II-induced AFs inflammatory response through PPAR $\gamma$ independent SOCS3/JAK/STAT signaling pathway.

CYP2J2 gene delivery upregulated aortic CYP2J2 expression and increased circulating and vascular EETs levels in mice

As described previously [13], a recombinant adeno-associated viral vector that expresses CYP2J2 (rAAV-CYP2J2) was generated to infect mice with viral titer at $1 \times 10^{11} \mathrm{pfu}$. An empty recombinant adeno-associated viral vector GFP (rAAV-GFP) was used as control. No obvious toxicity was observed. As expected, there was no CYP2J2 expression in aorta of wild type mouse. 4 weeks after rAAV-CYP2J2 vectors injection via tail veins, we observed abundant CYP2J2 expression in different layers of the aortic wall, especially adventitia and intima (Fig. 7A). These results were in consistent with those gained by western blot. Additionally, LC-MS 
Fig. 9. The potential mechanisms of EETs on adventitial remodeling induced by Ang II. The beneficial effects of 11,12-EET were associated with attenuating adventitial inflammatory response mediated by PPAR $\gamma$ dependent NF- $\kappa$ B and PPAR $\gamma$ independent SOCS3/JAK/STAT signaling pathways.

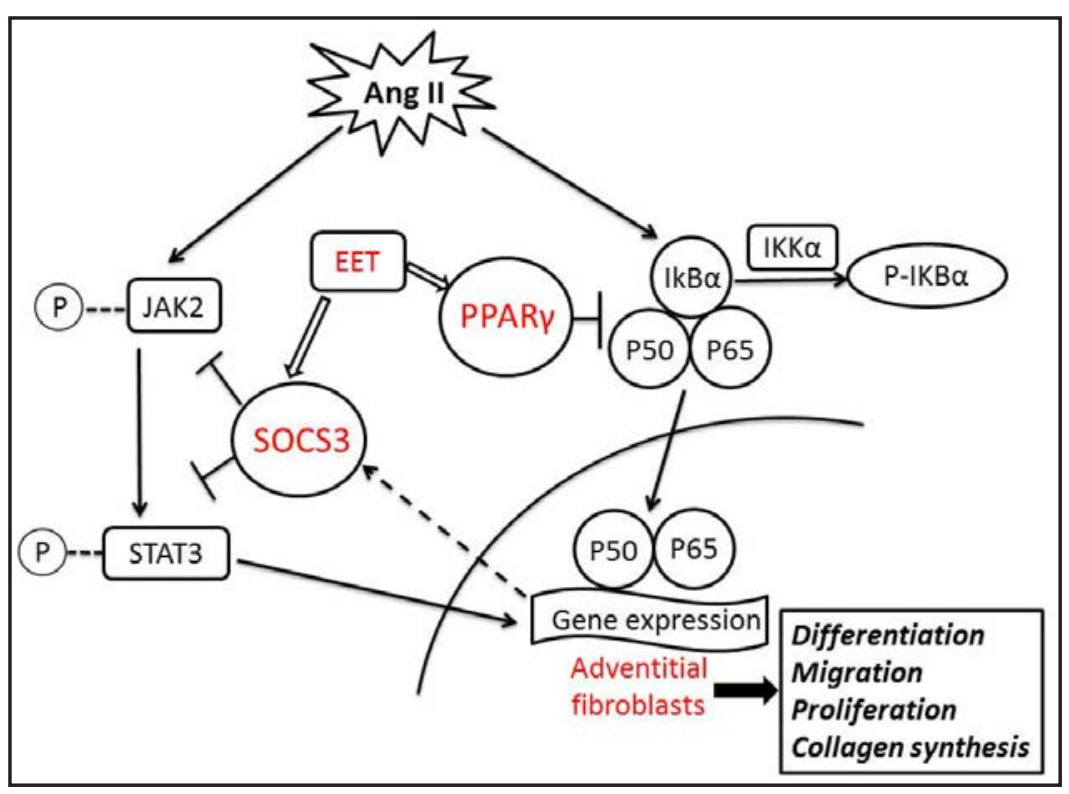

results showed a significant elevation in both vascular 11,12-, 14,15-EET and 11,12-, 14,15DHET levels were observed after CYP2J2 gene delivery (Fig. 7B). Similar results were found in vascular and circulating EETs level determined by using ELISA kits. These data indicated that adventitial CYP2J2 gene delivery was established successfully in animal models. We also analyzed the value of EETs to DHETs ratio and found it declined in Ang II + GFP and Ang II $+2 \mathrm{~J} 2$ group, compared with GFP and 2J2 group, respectively. It was speculated that Ang IIinduced sEH enzyme increase in mouse aorta contributed to the regulation of EET to DHET ratio.

\section{CYP2J2 overexpression suppresses Ang II-induced hypertension}

Previous study indicated that CYP2J2 overexpression reduced blood pressure in SHR [9], and lowing blood pressure contributed to attenuated vascular remodeling [36]. To clarify the direct role of CYP2J2 overexpression on adventitia, hydralazine was used to exclude the blood pressure-lowing effect on adventitial remodeling. As shown in Fig. 7C, systolic blood pressure (SBP) began to increase after Ang II infusion, and continued to rise to $156.3 \mathrm{mmHg}$ at 2 weeks. As expected, rAAV-CYP2J2 gene delivery prevented Ang II-induced increase in SBP level $(114.7 \mathrm{mmHg})(\mathrm{p}<0.05)$, which was not significantly different from that of Ang II + Hydralazine group $(117.7 \mathrm{mmHg})$ at an optimal dose $(250 \mathrm{mg} / \mathrm{L})$. As hydralazine reduced SBP level equal to CYP2J2 overexpression group, it provided an approach to investigate the individual effect of CYP2J2 gene delivery on adventitial remodeling.

\section{CYP2J2 overexpression suppresses Ang II-induced adventitial remodeling}

$\alpha$-SMA is the marker of AFs phenotypic transformation [31]. Ang II infusion led to an obvious increase in $\alpha$-SMA expression in adventitia, while CYP2J2 gene delivery prevented this effect with negative staining in adventitia, but only positive in media (Fig. 7D). Moreover, AFs proliferation marker PCNA expression was detected in aortic adventitia as shown in Fig. 7D. Our results indicated that Ang II infusion led to an obvious increase in PCNA expression in adventitia, while CYP2J2 gene delivery prevented this effect. CYP2J2 overexpression also reduced vessel wall thickening and collagen deposition in adventitia induced by Ang II infusion, which were determined by HE staining and Sirus Red staining. Meanwhile, as shown in Fig. 7D, hydralazine treatment also partially inhibited adventitial remodeling. However, CYP2J2 gene delivery showed better effects on adventitial remodeling than hydralazine group. Taken together, these results indicated that CYP2J2 overexpression exerted both blood pressure dependent and independent beneficial effects on adventitial remodeling. 


\section{Cellular Physiology Cell Physiol Biochem 2016;39:721-739 \\ \begin{tabular}{c|c|c|} 
DOI: 10.1159/000445663 & $\begin{array}{l}\text { O } 2016 \text { The Author(s). Published by S. Karger AG, Basel } \\
\text { www.karger.com/cpb }\end{array}$
\end{tabular} \\ Zhou et al.: CYP2J2 Gene Delivery Attenuated Adventitial Remodeling}

CYP2J2 overexpression reduces Ang II-induced adventitial inflammation

Adventitial inflammation plays an essential role in the pathogenesis of vascular remodeling [7]. As expected, Ang II infusion increased nuclear NF- $\kappa \mathrm{B}$ p 65 expression and I $\kappa \mathrm{B} \alpha$ degradation of mouse aortas (Fig. 8A, Fig. 8B), as well as JAK2 and STAT3 phosphorylation in aortas (Fig. 8C), while CYP2J2 overexpression significantly attenuated these effects induced by Ang II infusion. Moreover, CYP2J2 gene delivery increased SOCS3 expression, which acted as a feedback regulator of JAK/STAT pathway (Fig. 8C). Importantly, hydralazine exerted no obviously regulatory effects on NF- $\kappa B$ and SOCS3/JAK/STAT signaling pathways. Furthermore, ELISA analysis showed that CYP2J2 overexpression suppressed the secretion of inflammatory cytokines IL-6 and MCP-1 induced by Ang II infusion (Fig. 8D). These data indicated that CYP2J2 overexpression remarkably inhibited adventitial remodeling via attenuated adventitial inflammatory response.

\section{Discussions}

In this study, the effects of exogenous EETs addition and CYP2J2 gene delivery on Ang II-induced adventitial remodeling were investigated in vitro and in vivo. We demonstrated that CYP/sEH system existed in aortic adventitia, and participated in adventitial remodeling process. Importantly, we provided evidence that 11,12-EET administration inhibited Ang II-induced AFs proliferation, migration, differentiation and collagen synthesis, and these beneficial effects were mediated by PPAR $\gamma$-dependent NF- $\kappa B$ and PPAR $\gamma$-independent SOCS3/JAK/STAT inflammatory signaling pathway. Moreover, CYP2J2 overexpression significantly attenuated Ang II-induced adventitial remodeling in blood pressure dependent and independent manner. These results suggested that increasing EETs by CYP2J2 gene delivery could be considered as a novel approach to attenuate adventitial remodeling.

EETs exert a beneficial role in the therapy of cardiovascular disorders, including hypertension [9], atherosclerosis [11, 12], and abdominal aortic aneurysm [13]. The metabolic balance of EETs is determined by CYP catalysis and sEH hydrolysis [8]. There is adventitial remodeling process in the aortas of patients with aortic dissections [37,38]. In our study, CYP epoxygenases and sEH were found to exist in adventitia. Moreover, the adventitia of patients with aortic dissection exerted CYP2J2 inhibition and SEH activation, which decreased aortic EETs level, compared with normal populations. These results indicated that CYP/sEH system played important roles in adventitial remodeling process, which partly elucidated the pathogenesis of vascular disease, and provided a novel therapeutic target for the treatment of vascular disease in clinical practice.

Type 2 rAAV serotype is widely used in gene therapy, which is characterized by nonpathogenic, broad range of infectivity, long and stable transgene expression [39]. Our laboratory has established a rAAV-mediated CYP gene delivery vector to increase EETs generation in heart, aorta, and liver [13, 40, 41]. Here, we used type 2 rAAV mediated CYP2J2 gene delivery led to abundant aortic CYP2J2 expression and elevation of circulating and vascular EETs level. The high level of EETs affected adventitial remodeling process.

Adventitial remodeling is characterized by AFs proliferation, migration, phenotypic transformation and extracellular matrix synthesis [7]. CYP2J2 enzyme converts arachidonic acid to all four EET regioisomers. In the present study, we found that 8,9-EET, 11,12-EET and 14,15-EET remarkably alleviated Ang II-induced AFs differentiation, and 11,12-EET exerted the higher efficiency. Besides, the anti-inflammatory effect of 11,12-EET was widely explored in various disease models, including endothelial dysfunction, lung ischemia/reperfusion injury, Crohn's disease, and obesity. According to our data and previous reports, we selected 11,12-EET as a representative in our cellular experiments in vitro. Other regioisomeric EETs may also contribute to the protective effects of CYP2J2 overexpression on adventitial remodeling, but it still needs to be further elucidated. Meanwhile, CYP2J2 overexpression markedly attenuated Ang II-induced aortic adventitial phenotypic transformation. Myofibroblasts are highly activated cells that exhibit proliferative and migratory properties. 


\section{Cellular Physiology Cell Physiol Biochem 2016;39:721-739 \\ and Biochemistry Published online: July 27, $2016 \quad \begin{aligned} & \text { DOI: 10.1159/000445663 } 2016 \text { The Author(s). Published by S. Karger AG, Basel } \\ & \text { www.karger.com/cpb }\end{aligned}$ \\ Zhou et al.: CYP2J2 Gene Delivery Attenuated Adventitial Remodeling}

Previous research indicated that sEH inhibitor markedly inhibited fibroblasts proliferation and migration in cardiac fibrosis post-myocardial infarction [42]. In this study, we found 11,12-EET inhibited AFs proliferation and migration via PPAR $\gamma$-dependent NF- $\kappa B$ and PPAR $\gamma$-independent SOCS3/JAK/STAT inflammatory signaling pathways. Vascular fibrosis is one of the major changes in vascular diseases. Imbalance between ECM synthesis and degradation determines its abundance in vasculature [30]. Collagen Type I and Type III are the major vascular collagen, while mechanically stable collagen type I accumulates in the pathological process, and plays an important role during vascular remodeling [43]. Ang II participated in the fibrotic process during vascular damage, and adventitia was the main collagen deposition site in aortic wall [30]. CYP/sEH system was found to be involved in the process of fibrosis in various vascular abnormalities $[44,45]$. In our experiment, Ang II incubation increased collagen I expression in AFs, and 11,12-EET partially abolished this effect via PPAR $\gamma$ activation and SOCS3 induction. Meanwhile, CYP2J2 overexpression reduced adventitial collagen deposition in vivo. Taken together, our data demonstrated that CYP2J2 overexpression prevented Ang II-induced adventitial remodeling through PPAR $\gamma-\mathrm{NF}-\kappa \mathrm{B}$ and SOCS3/JAK/STAT signaling pathways. There are many other types of stimulus which could induce adventitial remodeling, such as TGF- $\beta$, hypoxia, hypertension, and adventitial inflammation. We also found 11,12-EET inhibited TGF- $\beta$-induced adventitial fibroblasts differentiation and migration (data not shown). It is speculated that CYP2J2-derived EETs attenuated TGF- $\beta$-induced adventitial remodeling. Growing evidences suggest that DHETs have biological activity in some systems. 11,12- and 14,15-DHET can hyperpolarize vascular smooth muscle [46] and endothelial cells [47] via activating $\mathrm{Ca}^{2+}$-activated $\mathrm{K}^{+}$channels, and enhancing the channel sensitivities to $\mathrm{Ca}^{2+}$ and voltage. In this study, we focused on EETs' effect, but did not evaluate the effect of DHETs on adventitial remodeling. We will design a more detailed experiment to explore the effects and mechanism of DHETs on adventitial remodeling in the near future.

It has been documented that aortic AFs participated in Ang II-induced vascular wall inflammation and remodeling [6, 7]. EETs may be PPAR $\gamma$ ligand [16], and PPAR $\gamma$ agonists attenuated Ang II-induced inflammation via NF- $\kappa B$ pathway [48]. Additionally, EETs exerted anti-inflammatory effect via inhibiting I $\kappa \mathrm{B} \alpha$ degradation and NF- $\kappa \mathrm{B}$ translocation [15]. Our experiments suggested a crucial role of PPAR $\gamma-\mathrm{NF}-\kappa \mathrm{B}$ pathway in the anti-inflammatory effect of exogenous EETs administration and CYP2J2 overexpression. 11,12-EET inhibited Ang II-

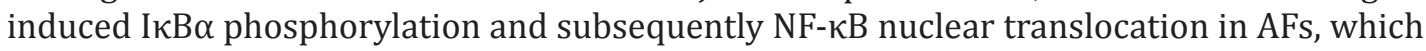
can be partially abolished by PPAR $\gamma$ antagonist GW9662. These results were consistent with previous study $[13,49]$. However, different cell types or treatment may cause heterogeneity of our work and previous data from Cui et al. [50] and Choudhary et al. [51] that Ang II causes minimal IkB degradation and is probably not responsible for p65 translocation. Furthermore, AFs secreted numerous inflammatory cytokines and chemokines to accelerate vascular remodeling, especially IL-6 and MCP-1 [6, 37]. CYP2J2 gene delivery and 11,12EET adminstration were found to abolish Ang II-induced IL-6 and MCP-1 production in vivo and in vitro. However, while 11,12-EET exerted regulatory effect on PPAR $\alpha$ activity, PPAR $\alpha$ antagonist GW6471 did not reverse Ang II effect on AFs differentiation and migration, and 11,12-EET displayed no linking with PPAR $\delta$. These results indicated that CYP2J2 overexpression inhibited Ang II-induced inflammatory response via PPAR $\gamma$-NF- $\kappa B$ signaling pathways, rather than PPAR $\alpha$ and PPAR $\delta$ related manner.

Abundant researches concerning the anti-inflammatory mechanism of EETs are based on PPAR $\gamma$-NF- $\kappa B$ pathway. However, there may be another novel underlying mechanism, as GW9662 pretreatment did not reverse the effect of EETs completely. Previous data indicated that JAK/STAT signaling pathway regulated inflammatory processes in various vascular cells, and Ang II activated this signaling pathway in cardiovascular diseases [52]. Meanwhile, JAK/ STAT signaling pathway regulated cells proliferation, migration and survival [53]. Our present study showed that 11,12-EET attenuated Ang II-induced JAK2/STAT3 phosphorylation, followed by phosphor-STAT3 nuclear translocation. These results were not consistent with that 14,15-EET stimulated the tyrosine phosphorylation and nuclear translocation of STAT-3 


\section{Cellular Physiology Cell Physiol Biochem 2016;39:721-739 $\begin{array}{lll}\text { DOI: 10.1159/000445663 } & \text { O 2016 The Author(s). Published by S. Karger AG, Basel } \\ \text { www.karger.com/cpb }\end{array}$ \\ Zhou et al.: CYP2J2 Gene Delivery Attenuated Adventitial Remodeling}

in human dermal microvascular endothelial cells [54]. It is speculated that the differences in cell type and treatment duration contributed to these inconsistence. Interestingly, PPAR $\gamma$ antagonist GW9662 pretreatment had no obvious impact on the effect of 11,12-EET on Ang II-induced JAK2/STAT3 phosphorylation, which indicated that 11,12-EET regulated JAK2/ STAT3 signaling pathway in PPAR $\gamma$-independent manner. These observations were consistent with the view of Ji et al. in monocyte [55]. SOCS3 is a negative feedback regulator of JAK/ STAT signaling pathway [34]. Interestingly, we found 11,12-EET and CYP2J2 gene delivery upregulated SOCS3 expression, thus inhibited JAK2/STAT3 inflammatory signaling pathway activation. As SOCS3 silence not only abolished the effects of EETs, but also alleviated the negative feedback regulation on Ang II-induced JAK/STAT activation, SOCS3 siRNA slightly reinforced Ang II-induced differentiation, proliferation, migration and collagen synthesis in AFs. These results were consistent with previous study in pancreatic acinar cells [18]. Moreover, SOCS3 silence reversed the effect of EETs on JAK/STAT pathway. Further studies are needed to evaluate the mechanism of EETs induced SOCS3 expression. Our results indicated that CYP2J2 overexpression reduced Ang II-induced inflammatory response via PPAR $\gamma$ independent SOCS3/JAK/STAT signaling pathway.

It has been reported that lowing blood pressure partially contributed to attenuate vascular remodeling $[56,57]$. As expected, Ang II infusion elevated systolic blood pressure (SBP) in C57BL/6J mice, and CYP2J2 overexpression prevented this hypertensive effect, which was in consistent with previous study in SHR [9]. It confused us for the antihypertensive effect or direct action of CYP2J2 overexpression on adventitia. In this study, hydralazine, as a vasodilator directly-act in vascular smooth muscle cells of arteries and arterioles [58], was used to exert an equal anti-hypertensive effect as CYP2J2 group [26, 58], which can exclude blood pressure lowering effect of CYP2J2 overexpression on adventitial remodeling process. Interestingly, we found that even though hydralazine treatment partially attenuated Ang II induced adventitial remodeling, but importantly CYP2J2 gene delivery exerted better protective effects than hydralazine. Furthermore, CYP2J2 overexpression, rather than hydralazine inhibited NF- $\mathrm{BB}$ and SOCS3/JAK/STAT inflammatory signaling pathways. In conclusion, these data suggested that the protective effect of CYP2J2 overexpression on Ang II induced adventitial remodeling was mediated by blood pressure dependent and independent manner.

In summary, the present study provided evidences that rAAV-CYP2J2 gene delivery remarkably attenuated Ang II-induced adventitial remodeling in vitro and in vivo. The beneficial effects were associated with attenuated adventitial inflammatory response mediated by PPAR $\gamma$ dependent NF- $\kappa B$ and PPAR $\gamma$ independent SOCS3/JAK/STAT signaling pathways as shown in Fig. 9. These results provided evidence that CYP2J2-derived EETs may serve as a novel therapeutic strategy for adventitial remodeling related diseases.

\section{Acknowledgments}

We thank Dr. Sandip Chaugai for English editing. This work was supported by fundings from the Natural Science Foundation Committee Projects (NOS.81100085 and 31130031).

\section{Disclosure Statement}

The authors have no conflict of interests to declare.

\section{References}

1 Heeneman S, Sluimer JC, Daemen MJ: Angiotensin-converting enzyme and vascular remodeling. Circ Res 2007;101:441-454. 


\section{Cellular Physiology Cell Physiol Biochem 2016;39:721-739 \begin{tabular}{l|l} 
DOI: 10.1159/000445663 & $\begin{array}{l}\text { O 2016 The Author(s). Published by S. Karger AG, Basel } \\
\text { www.karger.com/cpb }\end{array}$
\end{tabular} \\ Zhou et al.: CYP2J2 Gene Delivery Attenuated Adventitial Remodeling}

2 Stenmark KR, Nozik-Grayck E, Gerasimovskaya E, Anwar A, Li M, Riddle S, Frid M: The adventitia: Essential role in pulmonary vascular remodeling. Compr Physiol 2011;1:141-161.

3 An SJ, Liu P, Shao TM, Wang ZJ, Lu HG, Jiao Z, Li X, Fu JQ: Characterization and functions of vascular adventitial fibroblast subpopulations. Cell Physiol Biochem 2015;35:1137-1150.

4 Simpkins AN, Rudic RD, Roy S, Tsai HJ, Hammock BD, Imig JD: Soluble epoxide hydrolase inhibition modulates vascular remodeling. Am J Physiol Heart Circ Physiol 2010;298:H795-806.

5 Stenmark KR, Yeager ME, El Kasmi KC, Nozik-Grayck E, Gerasimovskaya EV, Li M, Riddle SR, Frid MG: The adventitia: Essential regulator of vascular wall structure and function. Annu Rev Physiol 2013;75:23-47.

6 Tieu BC, Ju X, Lee C, Sun H, Lejeune W, Recinos A, 3rd, Brasier AR, Tilton RG: Aortic adventitial fibroblasts participate in angiotensin-induced vascular wall inflammation and remodeling. J Vasc Res 2011;48:261272.

7 Maiellaro K, Taylor WR: The role of the adventitia in vascular inflammation. Cardiovasc Res 2007;75:640648.

8 Xu X, Zhang XA, Wang DW: The roles of cyp450 epoxygenases and metabolites, epoxyeicosatrienoic acids, in cardiovascular and malignant diseases. Adv Drug Deliv Rev 2011;63:597-609.

9 Xiao B, Li X, Yan J, Yu X, Yang G, Xiao X, Voltz JW, Zeldin DC, Wang DW: Overexpression of cytochrome p450 epoxygenases prevents development of hypertension in spontaneously hypertensive rats by enhancing atrial natriuretic peptide. J Pharmacol Exp Ther 2010;334:784-794.

10 Zheng C, Wang L, Li R, Ma B, Tu L, Xu X, Dackor RT, Zeldin DC, Wang DW: Gene delivery of cytochrome p450 epoxygenase ameliorates monocrotaline-induced pulmonary artery hypertension in rats. Am J Respir Cell Mol Biol 2010;43:740-749.

11 Theken KN, Schuck RN, Edin ML, Tran B, Ellis K, Bass A, Lih FB, Tomer KB, Poloyac SM, Wu MC, Hinderliter AL, Zeldin DC, Stouffer GA, Lee CR: Evaluation of cytochrome p450-derived eicosanoids in humans with stable atherosclerotic cardiovascular disease. Atherosclerosis 2012;222:530-536.

12 Zhang LN, Vincelette J, Cheng Y, Mehra U, Chen D, Anandan SK, Gless R, Webb HK, Wang YX: Inhibition of soluble epoxide hydrolase attenuated atherosclerosis, abdominal aortic aneurysm formation, and dyslipidemia. Arterioscler Thromb Vasc Biol 2009;29:1265-1270.

13 Cai Z, Zhao G, Yan J, Liu W, Feng W, Ma B, Yang L, Wang JA, Tu L, Wang DW: Cyp2j2 overexpression increases eets and protects against angiotensin ii-induced abdominal aortic aneurysm in mice. J Lipid Res 2013;54:1448-1456.

14 Rosen ED, Spiegelman BM: Ppargamma : A nuclear regulator of metabolism, differentiation, and cell growth. J Biol Chem 2001;276:37731-37734.

15 Moshal KS, Zeldin DC, Sithu SD, Sen U, Tyagi N, Kumar M, Hughes WM, Jr., Metreveli N, Rosenberger DS, Singh M, Vacek TP, Rodriguez WE, Ayotunde A, Tyagi SC: Cytochrome p450 (cyp) 2j2 gene transfection attenuates mmp-9 via inhibition of nf-kappabeta in hyperhomocysteinemia. J Cell Physiol 2008;215:771781.

16 Liu Y, Zhang Y, Schmelzer K, Lee TS, Fang X, Zhu Y, Spector AA, Gill S, Morisseau C, Hammock BD, Shyy JY: The antiinflammatory effect of laminar flow: The role of ppargamma, epoxyeicosatrienoic acids, and soluble epoxide hydrolase. Proc Natl Acad Sci U S A 2005;102:16747-16752.

17 Park EJ, Park SY, Joe EH, Jou I: 15d-pgj2 and rosiglitazone suppress janus kinase-stat inflammatory signaling through induction of suppressor of cytokine signaling 1 (socs1) and socs3 in glia. J Biol Chem 2003;278:14747-14752.

$18 \mathrm{Yu} \mathrm{JH}, \mathrm{Kim} \mathrm{KH}, \mathrm{Kim} \mathrm{H}$ : Socs 3 and ppar-gamma ligands inhibit the expression of il- 6 and tgf-beta1 by regulating jak2/stat3 signaling in pancreas. Int J Biochem Cell Biol 2008;40:677-688.

19 Teng Z, Tang D, Zheng J, Woodard PK, Hoffman AH: An experimental study on the ultimate strength of the adventitia and media of human atherosclerotic carotid arteries in circumferential and axial directions. J Biomech 2009;42:2535-2539.

20 Zhu DL, Herembert T, Marche P: Increased proliferation of adventitial fibroblasts from spontaneously hypertensive rat aorta. J Hypertens 1991;9:1161-1168.

21 Sun J, Sui X, Bradbury JA, Zeldin DC, Conte MS, Liao JK: Inhibition of vascular smooth muscle cell migration by cytochrome p450 epoxygenase-derived eicosanoids. Circ Res 2002;90:1020-1027.

22 Chen W, Bao Y, Chen X, Burton J, Gong X, Gu D, Mi Y, Bao L: Mycobacterium tuberculosis pe25/ppe41 protein complex induces activation and maturation of dendritic cells and drives th2-biased immune responses. Med Microbiol Immunol 2016;205:119-131. 


\section{Cellular Physiology Cell Physiol Biochem 2016;39:721-739 \begin{tabular}{l|l} 
DOI: 10.1159/000445663 & $\begin{array}{l}\text { O 2016 The Author(s). Published by S. Karger AG, Basel } \\
\text { www.karger.com/cpb }\end{array}$
\end{tabular} \\ Zhou et al.: CYP2J2 Gene Delivery Attenuated Adventitial Remodeling}

23 Chen K, Chen J, Li D, Zhang X, Mehta JL: Angiotensin ii regulation of collagen type i expression in cardiac fibroblasts: Modulation by ppar-gamma ligand pioglitazone. Hypertension 2004;44:655-661.

24 Drittanti L, Rivet C, Manceau P, Danos O, Vega M: High throughput production, screening and analysis of adeno-associated viral vectors. Gene Ther 2000;7:924-929.

25 Fukui T, Ishizaka N, Rajagopalan S, Laursen JB, Capers Qt, Taylor WR, Harrison DG, de Leon H, Wilcox JN, Griendling KK: P22phox mrna expression and nadph oxidase activity are increased in aortas from hypertensive rats. Circ Res 1997;80:45-51.

26 Inanaga K, Ichiki T, Matsuura H, Miyazaki R, Hashimoto T, Takeda K, Sunagawa K: Resveratrol attenuates angiotensin ii-induced interleukin-6 expression and perivascular fibrosis. Hypertens Res 2009;32:466471.

27 Robbins N, Thompson A, Mann A, Blomkalns AL: Isolation and excision of murine aorta; a versatile technique in the study of cardiovascular disease. J Vis Exp 2014:e52172.

28 Xu X, Zhao CX, Wang L, Tu L, Fang X, Zheng C, Edin ML, Zeldin DC, Wang DW: Increased cyp2j3 expression reduces insulin resistance in fructose-treated rats and db/db mice. Diabetes 2010;59:997-1005.

29 He Z, Zhang X, Chen C, Wen Z, Hoopes SL, Zeldin DC, Wang DW: Cardiomyocyte-specific expression of cyp $2 \mathrm{j} 2$ prevents development of cardiac remodelling induced by angiotensin ii. Cardiovasc Res 2015;105:304-317.

30 Che ZQ, Gao PJ, Shen WL, Fan CL, Liu JJ, Zhu DL: Angiotensin ii-stimulated collagen synthesis in aortic adventitial fibroblasts is mediated by connective tissue growth factor. Hypertens Res 2008;31:1233-1240.

31 Shen WL, Gao PJ, Che ZQ, Ji KD, Yin M, Yan C, Berk BC, Zhu DL: Nad(p)h oxidase-derived reactive oxygen species regulate angiotensin-ii induced adventitial fibroblast phenotypic differentiation. Biochem Biophys Res Commun 2006;339:337-343.

32 Di J, Jiang L, Zhou Y, Cao H, Fang L, Wen P, Li X, Dai C, Yang J: Ets-1 targeted by microrna-221 regulates angiotensin ii-induced renal fibroblast activation and fibrosis. Cell Physiol Biochem 2014;34:1063-1074.

33 Wray JA, Sugden MC, Zeldin DC, Greenwood GK, Samsuddin S, Miller-Degraff L, Bradbury JA, Holness MJ, Warner TD, Bishop-Bailey D: The epoxygenases cyp2j2 activates the nuclear receptor pparalpha in vitro and in vivo. PLoS One 2009;4:e7421.

34 Shi J, Wei L: Regulation of jak/stat signalling by socs in the myocardium. Cardiovasc Res 2012;96:345-347.

35 Tomita S, Ishibashi K, Hashimoto K, Sugino T, Yanagida T, Kushida N, Shishido K, Aikawa K, Sato Y, Suzutani T, Yamaguchi O: Suppression of socs3 increases susceptibility of renal cell carcinoma to interferon-alpha. Cancer Sci 2011;102:57-63.

36 Fernandes-Santos C, de Souza Mendonca L, Mandarim-de-Lacerda CA: Favorable cardiac and aortic remodeling in olmesartan-treated spontaneously hypertensive rats. Heart Vessels 2009;24:219-227.

37 Tieu BC, Lee C, Sun H, Lejeune W, Recinos A, 3rd, Ju X, Spratt H, Guo DC, Milewicz D, Tilton RG, Brasier AR: An adventitial il-6/mcp1 amplification loop accelerates macrophage-mediated vascular inflammation leading to aortic dissection in mice. J Clin Invest 2009;119:3637-3651.

38 Wang Z, Ren Z, Hu Z, Hu X, Zhang H, Wu H, Zhang M: Angiotensin-ii induces phosphorylation of erk1/2 and promotes aortic adventitial fibroblasts differentiating into myofibroblasts during aortic dissection formation. J Mol Histol 2014;45:401-412.

39 Lipiec A, Malecki M, Hajdukiewic K: [serotypes of adeno-associated viruses]. Postepy Biochem 2009;55:95102.

40 Dai M, Wu L, He Z, Zhang S, Chen C, Xu X, Wang P, Gruzdev A, Zeldin DC, Wang DW: Epoxyeicosatrienoic acids regulate macrophage polarization and prevent lps-induced cardiac dysfunction. J Cell Physiol 2015;230:2108-2119.

41 Li R, Xu X, Chen C, Wang Y, Gruzdev A, Zeldin DC, Wang DW: Cyp2j2 attenuates metabolic dysfunction in diabetic mice by reducing hepatic inflammation via the ppargamma. Am J Physiol Endocrinol Metab 2015;308:E270-282.

42 Sirish P, Li N, Liu JY, Lee KS, Hwang SH, Qiu H, Zhao C, Ma SM, Lopez JE, Hammock BD, Chiamvimonvat $\mathrm{N}$ : Unique mechanistic insights into the beneficial effects of soluble epoxide hydrolase inhibitors in the prevention of cardiac fibrosis. Proc Natl Acad Sci U S A 2013;110:5618-5623.

43 Zhang J, Fang NY, Gao PJ, Wu LY, Han WQ, Guo SJ, Shen WL, Zhu DL: Peroxisome proliferator-activated receptor-gamma agonists attenuate angiotensin ii-induced collagen type i expression in adventitial fibroblasts. Clin Exp Pharmacol Physiol 2008;35:72-77. 


\section{Cellular Physiology Cell Physiol Biochem 2016;39:721-739 \begin{tabular}{l|l} 
DOI: 10.1159/000445663 & $\begin{array}{l}\text { O 2016 The Author(s). Published by S. Karger AG, Basel } \\
\text { www.karger.com/cpb }\end{array}$
\end{tabular} \\ Zhou et al.: CYP2J2 Gene Delivery Attenuated Adventitial Remodeling}

44 Li L, Li N, Pang W, Zhang X, Hammock BD, Ai D, Zhu Y: Opposite effects of gene deficiency and pharmacological inhibition of soluble epoxide hydrolase on cardiac fibrosis. PLoS One 2014;9:e94092.

45 Zhao G, Tu L, Li X, Yang S, Chen C, Xu X, Wang P, Wang DW: Delivery of aav2-cyp2j2 protects remnant kidney in the 5/6-nephrectomized rat via inhibition of apoptosis and fibrosis. Hum Gene Ther 2012;23:688-699.

46 Lu T, Katakam PV, VanRollins M, Weintraub NL, Spector AA, Lee HC: Dihydroxyeicosatrienoic acids are potent activators of $\mathrm{ca}(2+)$-activated $\mathrm{k}(+)$ channels in isolated rat coronary arterial myocytes. J Physiol 2001;534:651-667.

47 Campbell WB, Deeter C, Gauthier KM, Ingraham RH, Falck JR, Li PL: 14,15-dihydroxyeicosatrienoic acid relaxes bovine coronary arteries by activation of $\mathrm{k}$ (ca) channels. Am J Physiol Heart Circ Physiol 2002;282:H1656-1664.

48 Wei-guo Z, Hui Y, Shan L, Yun Z, Wen-cheng N, Fu-lin Y, Fang-yan F, Jun-hua G, Jian-hua Z: Ppar-gamma agonist inhibits ang ii-induced activation of dendritic cells via the mapk and nf-kappab pathways. Immunol Cell Biol 2010;88:305-312.

49 Wolf G, Wenzel U, Burns KD, Harris RC, Stahl RA, Thaiss F: Angiotensin ii activates nuclear transcription factor-kappab through at1 and at2 receptors. Kidney Int 2002;61:1986-1995.

50 Cui R, Tieu B, Recinos A, Tilton RG, Brasier AR: Rhoa mediates angiotensin ii-induced phosphoser536 nuclear factor kappab/rela subunit exchange on the interleukin-6 promoter in vsmcs. Circ Res 2006;99:723-730.

51 Choudhary S, Lu M, Cui R, Brasier AR: Involvement of a novel rac/rhoa guanosine triphosphatase-nuclear factor-kappab inducing kinase signaling pathway mediating angiotensin ii-induced rela transactivation. Mol Endocrinol 2007;21:2203-2217.

52 Grote K, Luchtefeld M, Schieffer B: Janus under stress--role of jak/stat signaling pathway in vascular diseases. Vascul Pharmacol 2005;43:357-363.

53 Bromberg J, Darnell JE, Jr.: The role of stats in transcriptional control and their impact on cellular function. Oncogene 2000;19:2468-2473.

54 Cheranov SY, Karpurapu M, Wang D, Zhang B, Venema RC, Rao GN: An essential role for src-activated stat-3 in 14,15-eet-induced vegf expression and angiogenesis. Blood 2008;111:5581-5591.

55 Ji JD, Kim HJ, Rho YH, Choi SJ, Lee YH, Cheon HJ, Sohn J, Song GG: Inhibition of il-10-induced stat3 activation by 15-deoxy-delta12,14-prostaglandin j2. Rheumatology (Oxford) 2005;44:983-988.

56 Park JB, Schiffrin EL: Et(a) receptor antagonist prevents blood pressure elevation and vascular remodeling in aldosterone-infused rats. Hypertension 2001;37:1444-1449.

57 Pushpakumar SB, Kundu S, Metreveli N, Sen U: Folic acid mitigates angiotensin-ii-induced blood pressure and renal remodeling. PLoS One 2013;8:e83813.

58 Qi G, Jia L, Li Y, Bian Y, Cheng J, Li H, Xiao C, Du J: Angiotensin ii infusion-induced inflammation, monocytic fibroblast precursor infiltration, and cardiac fibrosis are pressure dependent. Cardiovasc Toxicol 2011;11:157-167. 